\title{
Stereorandomization as a Method to Probe Peptide Bioactivity
}

Thissa N. Siriwardena, Bee-Ha Gan, Thilo Köhler, Christian van Delden, Sacha Javor, and Jean-Louis Reymond*

Cite This: ACS Cent. Sci. 2021, 7, 126-134

Read Online

ACCESS |

Wll Metrics \& More

Article Recommendations

Supporting Information

ABSTRACT: Solid-phase peptide synthesis (SPPS) is usually performed with optically pure building blocks to prepare peptides as single enantiomers. Herein we report that SPPS using racemic amino acids provides stereorandomized (sr) peptides, containing up to billions of different stereoisomers, as well-defined single HPLC peaks, single mass products with high yield, which can be used to investigate peptide bioactivity. To exemplify our method, we show that stereorandomization abolishes the membranedisruptive effect of $\alpha$-helical amphiphilic antimicrobial peptides but preserves their antibiofilm effect, implying different mecha-

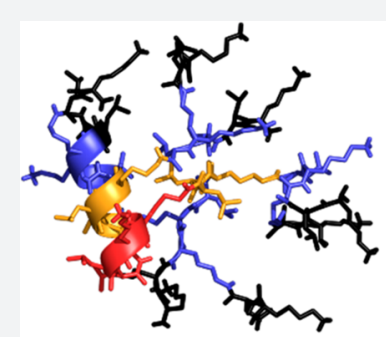

T25: $(\mathrm{KL})_{8}(\mathrm{KKL})_{4}(\mathrm{KLL})_{2} \mathrm{KKLL}$

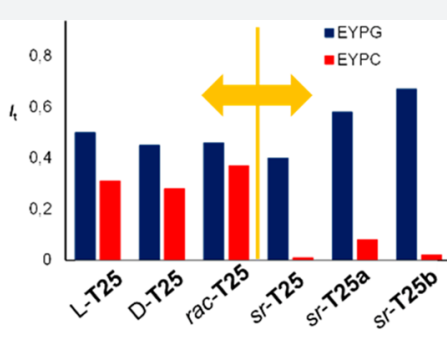

all L / all D stereorandom nisms involving folded versus disordered conformations. For antimicrobial peptide dendrimers by contrast, stereorandomization preserves antibacterial, membrane-disruptive, and antibiofilm effects but reduces hemolysis and cytotoxicity, thereby increasing their therapeutic index. Finally, we identify partially stereorandomized analogues of the last resort cyclic peptide antibiotic polymyxin B with preserved antibacterial activity but lacking membrane-disruptive and lipopolysaccharide-neutralizing activity, pointing to the existence of additional targets.

\section{INTRODUCTION}

The high efficiency of solid-phase peptide synthesis (SPPS) $)^{1,2}$ allows for interesting variations in synthesis planning, such as the split-and-mix protocol to prepare one-bead-one-compound libraries $^{3-5}$ or the synthesis of peptide mixtures by using several different amino acids in the same coupling step. ${ }^{6-8}$ Thanks to the availability of both $\mathrm{L}$ - and D-amino acids as synthetic building blocks, one can also vary residue stereochemistry and explore any number of stereoisomers of a given peptide sequence $^{9-11}$ or investigate mixtures of enantiomeric peptides synthesized individually as all $\mathrm{L}$ - or all $\mathrm{D}$ - sequences as reported for amyloid and hydrogel forming peptides. ${ }^{12-15}$ Here we asked the question whether SPPS using racemic rather than single enantiomer amino acids to form stereorandomized $(s r)$ peptides might provide new insights into the mechanism of action of peptides. As a case study, we focused on antimicrobial peptides (AMPs) ${ }^{16-19}$ antimicrobial peptide dendrimers (AMPDs), ${ }^{20-28}$ and polymyxin $\mathrm{B}^{29}$ which are all membrane-disruptive compounds active on Gram-negative bacteria including multidrug resistant strains.

\section{RESULTS AND DISCUSSION}

SPPS Provides sr-Peptides, sr-Peptide Dendrimers, and sr-Cyclic Peptides as Homogeneous Products. We first tested SPPS using racemic amino acids with AMP SB1, a short linear sequence of 13 residues, ${ }^{30}$ and with AMPD G3KL, which comprises 37 residues in a lysine-branched architecture accessible in 11 peptide coupling steps, to form $s r$-SB1 and $s r$ G3KL (Figure 1a). ${ }^{21}$ We also performed SPPS runs using 3:1 and $1: 3 \mathrm{~L} / \mathrm{D}$ ratios of each amino acid to form $s r^{3 / 1}-\mathrm{SB1}, s r^{3 / 1}$. G3KL, $s r^{1 / 3}-$ SB1, and $s r^{1 / 3}$-G3KL. To our delight, in each case, the crude as well as the HPLC-purified $s r$-product gave a single peak and a single mass indistinguishable from the homochiral parent compound when analyzed by LC/MS (Table 1, see also Supporting Information). Obtaining $s r$-peptides as homogeneous products is remarkable when considering that they contain a very large number of different stereoisomers but also reflects the fact that reversed-phase HPLC primarily separates compounds by hydrophobicity.

CD spectra of stereorandomized SB1 and G3KL in aqueous neutral buffer containing up to $20 \% \mathrm{v} / \mathrm{v}$ trifluoroethanol (TFE) as folding inducer ${ }^{31,32}$ showed an $\alpha$-helical signal with intensity proportional to the enantiomeric purity of the building blocks, resulting in a flat signal at $1: 1 \mathrm{D} / \mathrm{L}$ mixture of building blocks (Figure 1b,c, Figures S1 and S4). While the all L-enantiomers were susceptible to serum proteolysis (L-SB1: $t_{1 / 2} \sim 2 \mathrm{~h}, \mathrm{~L}-$ G3KL: $t_{1 / 2} \sim 15 \mathrm{~h}$ ), incorporation of as little as $25 \% \mathrm{D}$-amino acids in the $s r$-samples significantly reduced $\left(s r^{3 / 1}-\mathbf{S B 1}\right)$ or entirely blocked $\left(s r^{3 / 1}-\right.$ G3KL) serum proteolysis, reflecting the selectivity of proteases for L-peptides (Figure 1d).

Received: August 24, 2020

Published: January 19, 2021 
a)

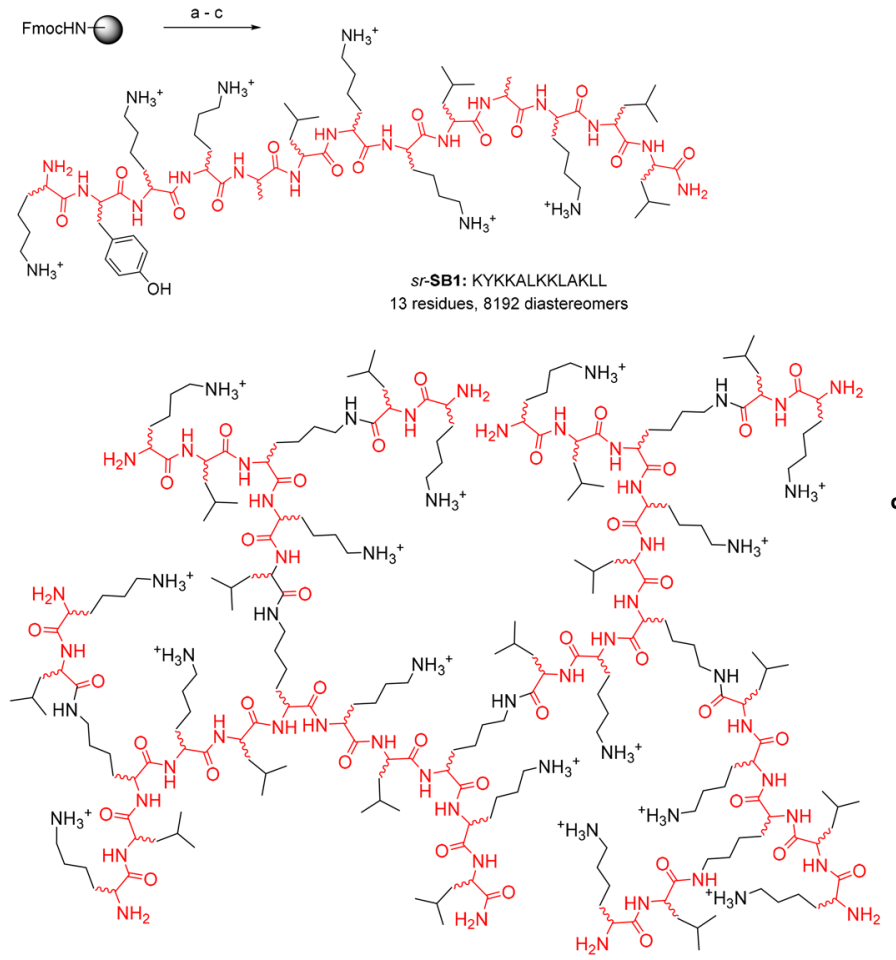

b)

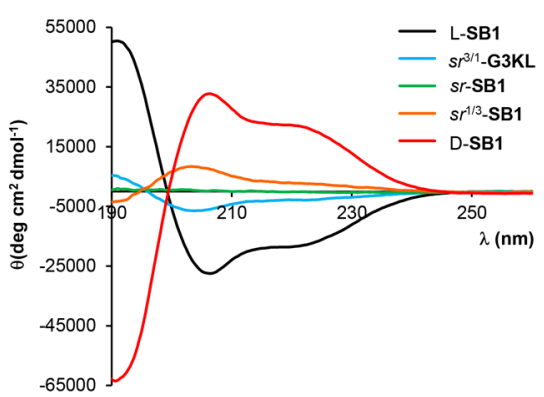

c)

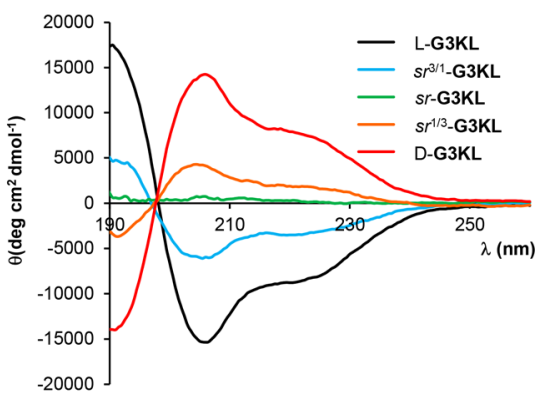

sr-G3KL: $(\mathrm{KL})_{8}(\mathrm{KKL})_{4}(\mathrm{KKL})_{2} \mathrm{KKL}$ 37 residues, $137,438,953,472$ diastereomers

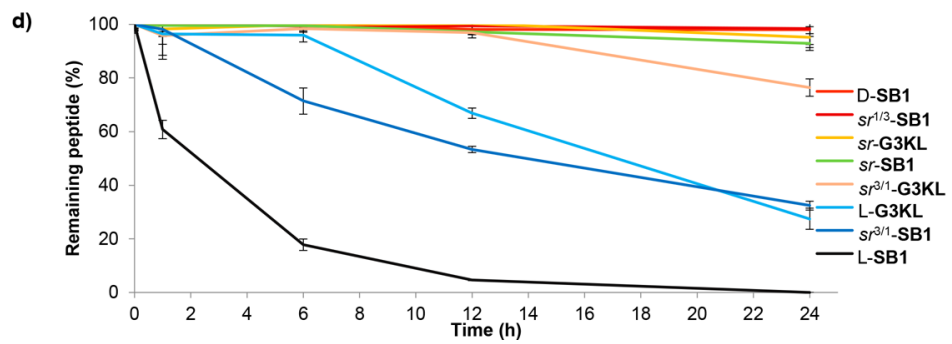

Figure 1. Synthesis of stereorandomized peptides and peptide dendrimers. (a) Stereorandomized SPPS of AMP sr-SB1 and AMPD sr-G3KL. SPPS conditions: (a) $20 \% \mathrm{v} / \mathrm{v}$ piperidine in DMF, $2 \mathrm{~min}, 25^{\circ} \mathrm{C}$ and $5 \mathrm{~min}, 50^{\circ} \mathrm{C}$; (b) FmocAAOH ( 5 equiv), Oxyma (7.5 equiv), DIC (10 equiv), 10 min, $50{ }^{\circ} \mathrm{C}$; (c) TFA/TIS/ $\mathrm{H}_{2} \mathrm{O}$ (94:5:1), $4 \mathrm{~h}$ at room temperature. (b) CD spectra of L-, D- and $s r$ - SB1 and (c) CD spectra of L-, D-, and $s r-G 3 \mathrm{KL}$, at $200 \mu \mathrm{g} / \mathrm{mL}$ TFA salt of the compound in $6 \mathrm{mM}$ aq. phosphate buffer $\mathrm{pH} 7.4$ with the $20 \%$ of TFE added. (d) Serum stability assay. Conditions: $400 \mu \mathrm{M}$ compound in aq. $0.1 \mathrm{M}$ Tris buffer $\mathrm{pH} 7.5$ containing $25 \% \mathrm{v} / \mathrm{v}$ human serum.

Stereorandomized SPPS of AMPs DJK5 (12 residues) ${ }^{33}$ and indolicidin (indo, 13 residues), ${ }^{34-37}$ AMPDs TNS18 (18 residues, 10 steps ${ }^{24}$ and T25 (38 residues, 12 steps), ${ }^{25}$ as well as polymyxin B2 (PMB2, 10 residues, cyclic peptide), ${ }^{38}$ similarly provided the corresponding $s r$-sequences as homogeneous products in excellent isolated yields, which showed similarly flat CD spectra (Figures S2, S3, S5, S6, and S7). We also obtained clean products when we stereorandomized only selected positions in the case of T25 and PMB2. Taken together, these different syntheses showed that partially or fully stereorandomized peptides are readily accessible in homogeneous form for linear, dendritic, and cyclic peptide sequences.

Stereorandomization Abolishes the Membrane-Disruptive and Antibacterial Activities of $\alpha$-Helical AMPs SB1 and DJK5 but Does Not Affect Their Biofilm Inhibition or the Activity of the Disordered AMP Indolicidin. AMPs SB1 (as the all L- or all D-enantiomer) folds to an amphiphilic $\alpha$-helix stable as 4-helix bundle within crystal structures as well as in a membrane environment. ${ }^{30}$
Stereorandomization of SB1 to form 1:3, 1:1, and 3:1 sr-SB1 entirely abolished its antimicrobial activity on Gram-negative strains (Pseudomonas aeruginosa, Acinetobacter baumannii, Klebsiella pneumoniae) or strongly reduced it (Escherichia coli) (Table 1, columns 5-8). The membrane-disruptive activity of SB1 on synthetic vesicles containing either egg white phosphatidyl glycerol (EYPG, mimicking bacterial membranes) or egg white phosphatidyl choline (EYPC, mimicking eukaryotic membranes) was similarly strongly reduced upon stereorandomization (Table 1, columns 9-10, Figure 2a, Figure S8). ${ }^{39}$ By contrast, the racemate rac-SB1, the 1:1 mixture of all $\mathrm{L}$ - and all D-enantiomers, was as active as the individual enantiomers in the different assays. None of the samples showed any measurable hemolysis on human erythrocytes (Table 1, column 11). We observed similar effects with AMP DJK5, ${ }^{33}$ which also forms an amphiphilic $\alpha$ helix (Figure S2) and essentially lost its antibacterial and EYPG vesicle leakage activity in its $s r$-version compared with the all L- or all D-enantiomers (Figure S9). 
Table 1. Synthesis and Activity of Homochiral (L- or D-), Stereorandomized (sr-) and Racemic (-rac) Antimicrobial Peptides, Peptide Dendrimers, and Polymyxin B

\begin{tabular}{|c|c|c|c|c|c|c|c|c|c|c|c|}
\hline \multirow[t]{2}{*}{ Cpd. ${ }^{a}$} & SPPS yield & MS analysis & $\begin{array}{c}\text { analytical } \\
\text { HPLC }\end{array}$ & $\begin{array}{c}P . \\
\text { aeruginosa }\end{array}$ & A.baumannii & E. coli & $\begin{array}{c}K . \\
\text { pneumoniae }\end{array}$ & $\begin{array}{l}\text { EYPG } \\
\text { vesicle } \\
\text { leakage }\end{array}$ & $\begin{array}{c}\text { EYPC } \\
\text { vesicle } \\
\text { leakage }\end{array}$ & $\begin{array}{c}\text { hemolysis } \\
\text { on hRBC, } \\
\text { MHC }\end{array}$ & $\begin{array}{l}\text { PAO1 } \\
\text { biofilm } \\
\text { MBIC }\end{array}$ \\
\hline & $\operatorname{mg}(\%)^{b}$ & calc. $/$ obs. $^{c}$ & $\underset{(m i n .)^{d}}{t_{\mathrm{R}}}$ & \multicolumn{4}{|c|}{$\operatorname{MIC}(\mu \mathrm{g} / \mathrm{mL})^{e}$} & $(\%)^{f}$ & $(\%)^{f}$ & $(\mu \mathrm{g} / \mathrm{mL})^{g}$ & $\begin{array}{l}(\mu \mathrm{g} / h \\
\mathrm{mL})^{\prime}\end{array}$ \\
\hline \multicolumn{12}{|c|}{ Linear Peptides } \\
\hline \multicolumn{12}{|c|}{ SB1: KYKKALKKLAKLL (13 residues, 8192 diastereomers) } \\
\hline L-SB 1 & $56(30)$ & $1543.07 / 1543.08$ & 1.53 & 2 & 2 & 0.5 & 32 & 60 & 52 & $>2000$ & 16 \\
\hline D-SB1 & $74(26)$ & $1543.07 / 1543.07$ & 1.55 & 1 & 2 & 0.5 & 32 & 70 & 45 & $>2000$ & 8 \\
\hline rac-SB1 & - & - & - & 2 & 2 & 1 & 16 & 91 & 51 & $>2000$ & 16 \\
\hline$s r$-SB 1 & $62(22)$ & $1543.07 / 1543.08$ & 1.53 & $>64$ & $>64$ & 32 & $>64$ & 12 & 1 & $>2000$ & 16 \\
\hline$s r^{3 / 1}-\mathbf{S B 1}$ & $48(17)$ & $1543.07 / 1543.07$ & 1.54 & $>64$ & $>64$ & 16 & $>64$ & 20 & 9 & $>2000$ & 16 \\
\hline$s r^{1 / 3}-$ SB1 & $57(20)$ & $1543.07 / 1543.07$ & 1.54 & $>64$ & $>64$ & 16 & $>64$ & 27 & 6 & $>2000$ & 16 \\
\hline \multicolumn{12}{|c|}{ DJK5: VQWRAIRVRVIR (12 residues, 4096 diastereomers) } \\
\hline L-DJK5 & $22(9)$ & $1549.98 / 1549.98$ & 1.53 & 32 & 16 & 32 & $>64$ & 32 & 1 & $>2000$ & $>32$ \\
\hline D-DJK5 & $19(7)$ & $1549.98 / 1549.98$ & 1.50 & $4-2$ & 8 & $4-2$ & 2 & 36 & 2 & 1000 & 8 \\
\hline$s r-\mathrm{DJK} 5$ & $13(8)$ & $1549.98 / 1549.98$ & 1.52 & $>64$ & $>64$ & 16 & $>64$ & 8 & 1 & $>2000$ & 32 \\
\hline \multicolumn{12}{|c|}{ Indolicidin: ILPWKWPWWPWRR (13 residues, 8192 diastereomers) } \\
\hline L-Indo & $28(10)$ & $1905.05 / 1905.04$ & 1.80 & $>64$ & 16 & 32 & 32 & 18 & 18 & 125 & $>32$ \\
\hline D-Indo & $22(8)$ & $1905.05 / 1905.05$ & 1.82 & $>64$ & 16 & 16 & 32 & 35 & 18 & 125 & $>32$ \\
\hline$s r$-Indo & $18(7)$ & $1905.05 / 1905.05$ & 1.87 & $>64$ & 8 & 16 & 16 & 18 & 37 & 125 & $>32$ \\
\hline
\end{tabular}

Peptide Dendrimers

G3KL: $(\mathrm{KL})_{8}(\mathrm{KKL})_{4}(\mathrm{KKL})_{2} \mathrm{KKL}$ (37 residues, 137438953472 possible diastereomers)

\begin{tabular}{|c|c|c|c|c|c|c|c|c|c|c|}
\hline L-G3KL & $124(23)$ & $4531.38 / 4531.43$ & 1.47 & 2 & 4 & 1 & $>64$ & 42 & 6 & 1000 \\
\hline D-G3KL & $142(17)$ & $4531.38 / 4531.37$ & 1.46 & 4 & 8 & 1 & 32 & 51 & 8 & 1000 \\
\hline$r a c-G 3 K L$ & - & - & - & 4 & 8 & 2 & $>64$ & 77 & 1 & 1000 \\
\hline$s r-\mathbf{G} 3 \mathrm{KL}$ & $138(24)$ & $4531.38 / 4531.43$ & 1.53 & 8 & $>64$ & 8 & $>64$ & 38 & 1 & 1000 \\
\hline$s r^{3 / 1}-$ G3KL & $94(11)$ & $4531.38 / 4531.43$ & 1.54 & 4 & $>64$ & 8 & $>64$ & 58 & 1 & 1000 \\
\hline$s r^{1 / 3}-\mathrm{G} 3 \mathrm{KL}$ & $86(10)$ & $4531.38 / 4531.44$ & 1.52 & 8 & $>64$ & 4 & $>64$ & 66 & 1 & 1000 \\
\hline \multicolumn{11}{|c|}{ NS18: $(\mathrm{OF})_{4}(\mathrm{KBL})_{2} \mathrm{KKLK}\left(\mathrm{C}_{10}\right)$ (18 residues, 262144 possible diastereomers $)$} \\
\hline L-TNS18 & $121(42)$ & $2395.61 / 2395.61$ & 1.64 & 2 & 2 & 4 & $>64$ & 64 & 1 & 1000 \\
\hline D-TNS18 & $156(14)$ & $2395.61 / 2395.60$ & 1.63 & 4 & 8 & 4 & $>64$ & 65 & 5 & 500 \\
\hline rac-TNS18 & - & - & - & 4 & 4 & 2 & $>64$ & 76 & 3 & 500 \\
\hline$s r$-TNS18 & $8(4)$ & $2395.61 / 2395.60$ & 1.64 & 4 & 8 & 4 & $>64$ & 61 & 3 & 1000 \\
\hline
\end{tabular}

T25: $(\mathrm{KL})_{8}(\mathrm{KKL})_{4}(\mathrm{KLL})_{2} \mathrm{KKLL}$ (38 residues, 274877906944 possible diastereomers)

\begin{tabular}{|c|c|c|c|c|c|c|c|c|c|c|c|}
\hline L-T25 & $26(7)$ & $4614.44 / 4614.47$ & 1.55 & 4 & 4 & 4 & 16 & 50 & 31 & 62.5 & 8 \\
\hline D-T25 & $57(7)$ & $4614.44 / 4614.45$ & 1.59 & 4 & 2 & 8 & 16 & 45 & 28 & 125 & 8 \\
\hline rac-T25 & - & - & - & 4 & 4 & 8 & 16 & 46 & 37 & 31.25 & 8 \\
\hline$s r-\mathbf{T} 25$ & $3854(23)$ & $4614.44 / 4614.44$ & 1.59 & 2 & 8 & 4 & 32 & 40 & 1 & $>2000$ & 8 \\
\hline \multicolumn{12}{|c|}{-T25a: $\left(\mathrm{KL}_{\underline{8}}(\mathrm{KKL})_{4}(\mathrm{KLL})_{2} \mathrm{KKLL}\right.$ (38 residues, G2 and G3 stereorandomized, 268435456 diastereomers) } \\
\hline & $34(6)$ & $4614.44 / 4614.45$ & 1.61 & 4 & 4 & 4 & 32 & 58 & 8 & 62.5 & $>16$ \\
\hline
\end{tabular}

Cyclic Peptides

polymyxin B ( 10 residues, 1024 possible diastereomers)

\begin{tabular}{|c|c|c|c|c|c|c|c|c|c|c|c|}
\hline PMB & - & - & - & 0.25 & 0.25 & 0.25 & 0.25 & 53 & 2 & $>2000$ & 8 \\
\hline PMB2 & $26(3)$ & $1188.73 / 1188.74$ & 1.58 & 0.25 & 0.25 & 0.25 & 0.5 & 39 & 1 & $>2000$ & 8 \\
\hline$s r-\mathrm{PMB2}$ & $148(14)$ & $1188.73 / 1188.74$ & 1.55 & 32 & 64 & 2 & $>64$ & 5 & 6 & $>2000$ & $>16$ \\
\hline \multicolumn{12}{|c|}{$s r$-PMB2a (10 residues, residues $1,3,4,7,9$, and 10 racemic, 64 diastereomers) } \\
\hline & $24(5)$ & $1188.73 / 1188.74$ & 1.56 & 4 & 4 & 1 & $>64$ & 1 & 1 & $>2000$ & 16 \\
\hline \multicolumn{12}{|c|}{$s r$-PMB2b (10 residues, residues $2,3,4,9$, and 10 racemic, 32 diastereomers) } \\
\hline & $18(3)$ & $1188.73 / 1188.74$ & 1.58 & 2 & 2 & 0.25 & 2 & 1 & 1 & $>2000$ & 16 \\
\hline
\end{tabular}

${ }^{a}$ One-letter codes for amino acids, $K=$ branching lysine L- and D- sequences are with only L- or only D-amino acids, $s r$-sequences have all positions individually racemized corresponding to the indicated number of diastereomers, and rac-sequences are a 1:1 mixture of all $\mathrm{L}$ - and all $\mathrm{D}$-sequences, in $s r$-T25a and $s r$-T25b only the residues underlined are racemic, see Figures 1 and 4 for structural formulas of AMPDs and PMB. ${ }^{b}$ Yields given for RP-HPLC purified product. ${ }^{c}$ High-resolution electrospray ionization mass spectrometry (positive mode), the calculated monoisotopic mass, and the observed mass in the reconstructed spectrum are given. ${ }^{d}$ Retention time in analytical RP-HPLC $(\mathrm{A} / \mathrm{D}=100 / 0$ to $0 / 100$ in 2.2 min, $\lambda=214$ $\mathrm{nm}$ ). ${ }^{e}$ Minimum inhibitory concentration (MIC, $\mu \mathrm{g} / \mathrm{mL}$ ) was determined on P. aeruginosa PAO1, A. baumannii ATCC 19606, E. coli W3110 and

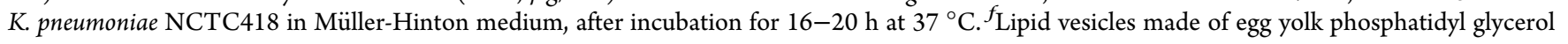


Table 1. continued

(EYPG) or egg yolk phosphatidyl choline (EYPC) were suspended in buffer (10 mM TRIS, $107 \mathrm{mM} \mathrm{NaCl}, \mathrm{pH} 7.4)$. After $50 \mathrm{~s}$, the indicated compound was added to reach the indicated concentration. After $300 \mathrm{~s}, 1.2 \%$ Triton X-100 was added for full fluorescein release. The percentage leakage observed with $10 \mu \mathrm{g} / \mathrm{mL}$ compound at $250 \mathrm{~s}$ is given. Full curves are given in Figures S8-S14. ${ }^{g}$ Minimum hemolytic concentration (MHC) measured on human red blood cells in $10 \mathrm{mM}$ phosphate, $150 \mathrm{mM} \mathrm{NaCl}, \mathrm{pH} \mathrm{7.4,25}{ }^{\circ} \mathrm{C}, 4 .{ }^{h}$ Minimum biofilm inhibitory concentration (MBIC, $\mu \mathrm{g} / \mathrm{mL}$ ) was measured in $0.25 \%(\mathrm{w} / \mathrm{v})$ nutrient broth no. 2, Oxoid on P. aeruginosa PAO1. All experiments were done in at least two independent duplicates, see the Supporting Information for details.
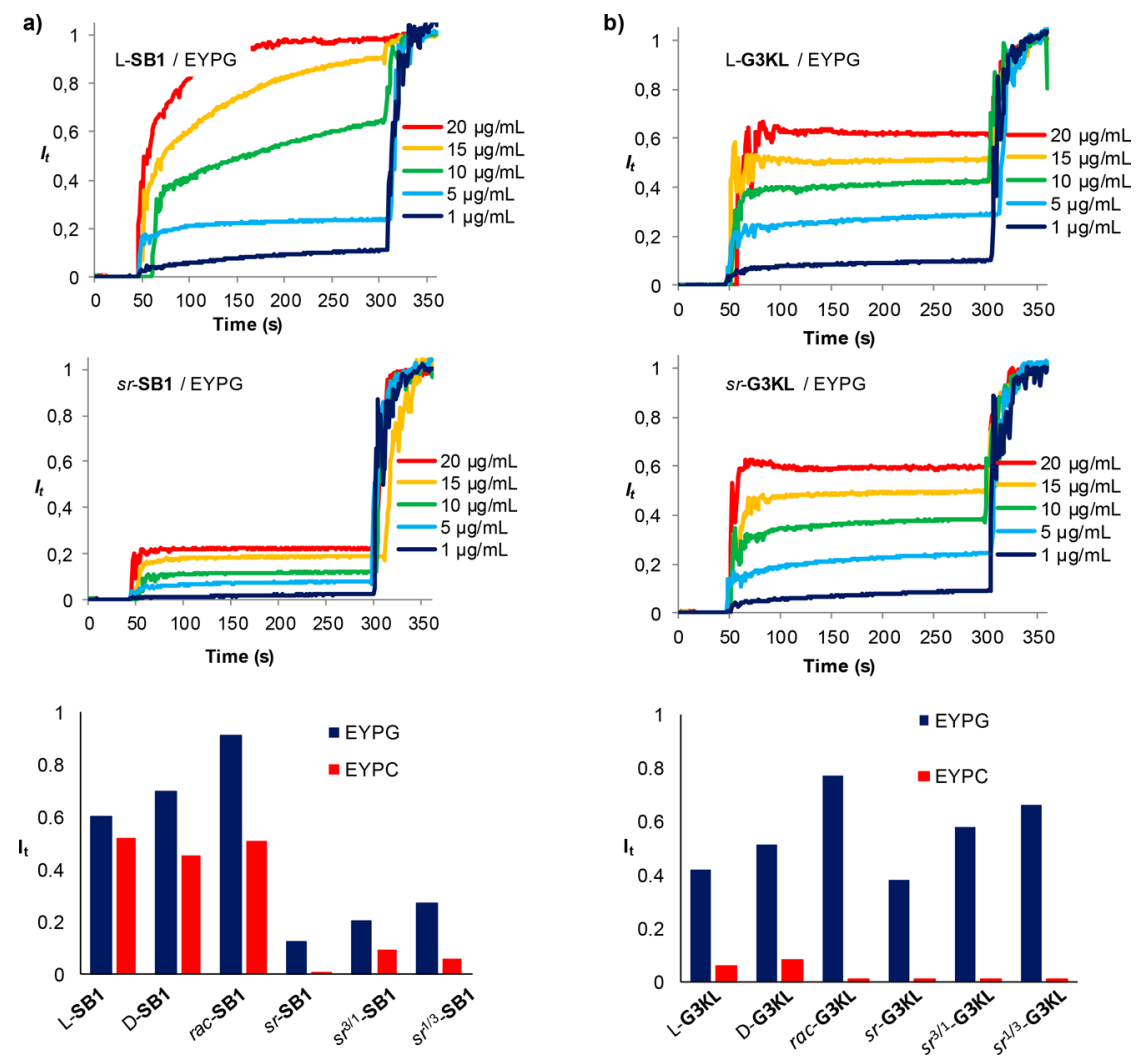

Figure 2. Vesicle leakage assays with homochiral and stereorandomized SB1 (a) and G3KL (b). Lipid vesicles made of egg yolk phosphatidyl

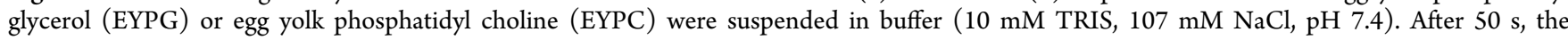
indicated compound was added to reach the indicated concentration. After $300 \mathrm{~s}, 1.2 \%$ Triton X-100 was added for full fluorescein release. The bar plots report relative fluorescence signal intensity measured after $250 \mathrm{~s}$ at $10 \mu \mathrm{g} / \mathrm{mL}$.

AMPs SB1 and DJK5 also exhibited a significant inhibitory activity against $P$. aeruginosa biofilms (Table 1 , column 12). In contrast to the antibacterial effect, however, biofilm inhibition by SB1 was independent of stereochemical purity. For DJK5, antibiofilm effects required the presence of D-residues, an effect which has been shown to protect this peptide from proteolysis. $^{33}$ By comparison, indolicidin (indo), ${ }^{34-37}$ for which $\mathrm{CD}$ spectroscopy indicates a disordered conformation (Figure S3), was more active as $s r$-indo than either L-indo or D-indo in terms of antibacterial and vesicle leakage activity (Figure S10). This data shows that indolicidin is membrane disruptive in a disordered conformation and does not require folding to be active. No antibiofilm activity was detected with any of the three indo peptides. Note that stereorandomization did not affect the hemolytic activity of any of the three linear AMPs discussed above, which was very weak for SB1 and DJK5 but quite significant for indo (Table 1, column 11).

Taken together, these experiments show that the antibacterial and membrane-disruptive activity of $\alpha$-helical AMPs SB1 and DJK5 requires $\alpha$-helical folding, while their antibiofilm activity does not and therefore must involve a disordered conformation. The AMP indo represents a useful control showing that membrane disruption is also possible for peptides with disordered conformation but in this case is unaffected by stereorandomization.

Stereorandomization of AMPDs G3KL and TNS18 Preserves Their Membrane-Disruptive, Antibacterial, and Antibiofilm Activities. AMPD G3KL exhibits an $\alpha$ helical CD spectrum in membrane-like environments similar to linear AMPs. ${ }^{21}$ Modeling studies with L-G3KL indicate partial $\alpha$-helical segments within the dendrimer, however without formation of a globally amphiphilic conformation. ${ }^{25}$ The same applies to TNS18, a smaller G2 analogue of G3KL optimized for activity by addition of a lipid tail at the core to enhance membrane interactions, and which also adopts an $\alpha$-helical but not globally amphiphilic conformation in a membrane-like environment. $^{24}$

Here we found that stereorandomization of AMPDs G3KL and TNS18 only very partially affected their antibacterial activity (Table 1, only G3KL lost activity against A. baumannii upon stereorandomization). Similarly, their membrane-disruptive activity as measured by vesicle leakage assays, their weak hemolytic activity, and their biofilm inhibition were all unaffected by stereorandomization (Figure 2b, Table 1, Figure 


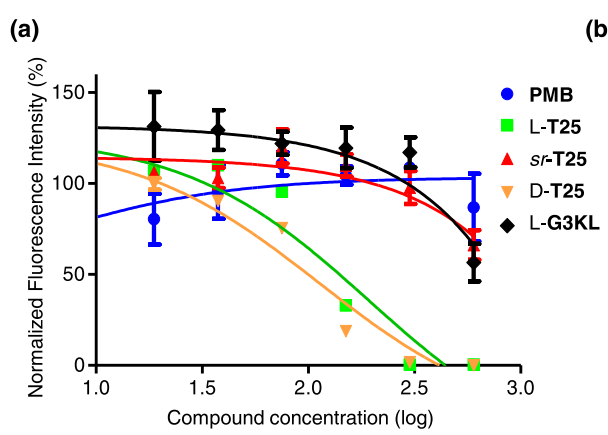

(b)

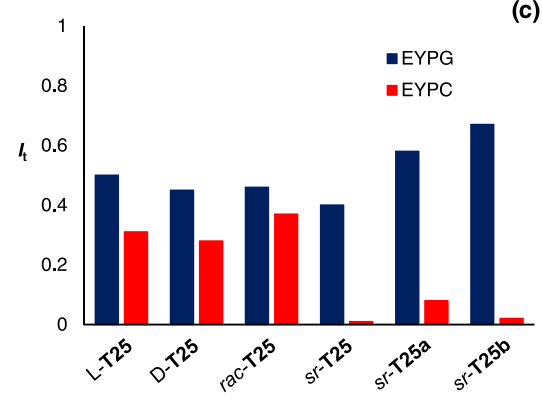

(d)

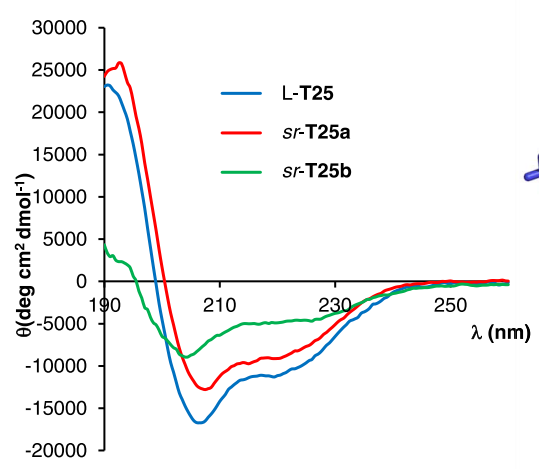

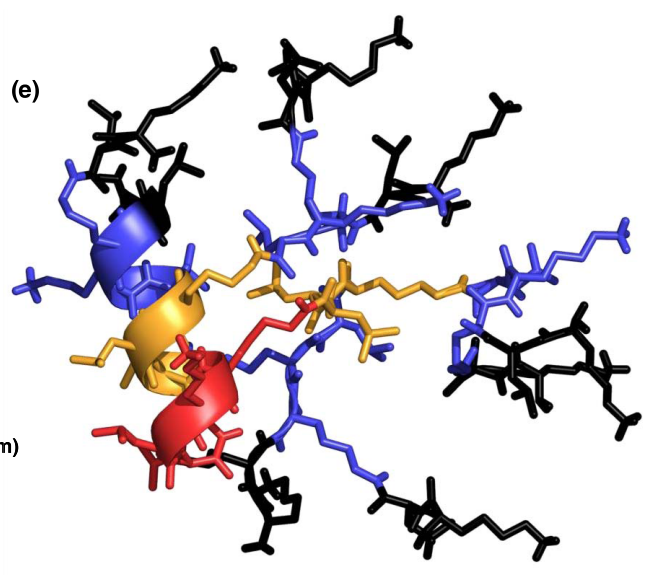

T25: $(\mathrm{KL})_{8}(K K L)_{4}(K L L)_{2} K K L L$

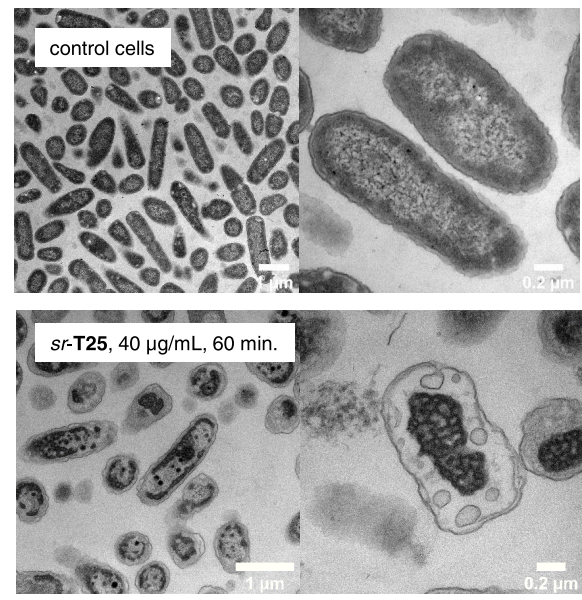

(f)

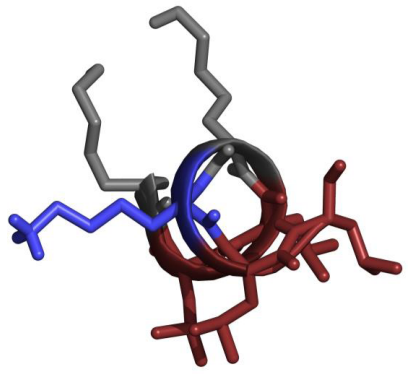

T25 G1-G0 core: KLLKKLL

Figure 3. Full and partial stereorandomization of AMPD T25. (a) Cytotoxicity of G3KL, polymyxin B, and T25 analogues in HepG2 cells in DMEM containing 10\% FBS. (b) Vesicle leakage assays. (c) TEM images of $P$. aeruginosa cells treated for 60 min at $10 \times$ MIC with $s r$-T25 (40 $\mu \mathrm{g} /$ $\mathrm{mL}$ ). (d) CD spectra of T25 analogues (TFA salt $200 \mu \mathrm{g} / \mathrm{mL}$ ) in aq. $6 \mathrm{mM}$ phosphate $\mathrm{pH} 7.4$ upon addition of $20 \%$ TFE. (e) Molecular dynamics simulation of dendrimer L-T25. Stick model of MD structures color-coded by dendrimer generation ( red = G0, orange = G1, blue = G2, black = G3) highlighting the $\alpha$-helical fold. (f) Cut-out view of (e) showing the G1-G0 $\alpha$-helical core of L-T25 (brown = leucine, blue = lysine, gray $=$ branching lysine).

S11 and S12). These data show that $\alpha$-helical folding is not required for these activities and support our previous hypothesis, based on modeling studies, that membrane disruption by AMPDs is independent of helical folding and is triggered by a transition from a hydrophobically collapsed conformation in water to an open conformation exposing hydrophobic residues in contact with the bacterial membrane. $^{25}$

Stereorandomization of AMDP T25 Increases Its Selectivity against Bacteria over Eukaryotic Cells. We next tested the effect of stereorandomization on AMPD T25, an analogue of G3KL with a more hydrophobic dendrimer core featuring one additional leucine residue in G0 (KL $\rightarrow$ $\mathrm{KLL}$ ) and a lysine-leucine mutation in G1 (KL $\rightarrow \mathrm{LL}$ ). Compared with G3KL, the modified dendrimer core of T25 results in a broader activity spectrum against Gram-negative bacteria also including Klebsiella pneumoniae, against which G3KL is inactive, but also induces strong hemolysis ( $\mathrm{MHC}=$ $62.5 \mu \mathrm{g} / \mathrm{mL}$, Table 1) and toxicity against various human cell lines (Figure 3a, Figure S15). ${ }^{25}$

Similar to the above AMPDs, stereorandomization to form $s r$-T25 preserved the vesicle leakage activity on anionic EYPG vesicles mimicking bacterial membranes (blue bars, Figure $3 \mathrm{~b}$, Figure S13). sr-T25 also displayed similar antibacterial activities as L- and D-T25 including a panel of MDR bacteria, except for K. pneumoniae NCTC418 and the P. aeruginosa clinical isolate ZEM9A (Table 1, Table S1). Transmission electron microscopy (TEM) images of $P$. aeruginosa cells exposed to $s r$-T25 showed extensive membrane damage, in line with its membrane-disruptive activity on EYPG vesicles (Figure 3c, Figure S16). However, stereorandomization of T25 entirely abolished its hemolysis and its vesicle leakage activity on neutral EYPC vesicles mimicking eukaryotic membrane (Table 1, Figure $3 \mathrm{~b}$, red bars). Furthermore, $s r$ T25 showed reduced cell toxicity compared to L- and D-T25 (Figure $3 \mathrm{a}$ and Figure S15).

Taken together, these data showed that stereorandomization can selectively affect membrane-disruptive properties leading to a higher selectivity for bacterial over eukaryotic membranes and a stronger activity against bacteria versus eukaryotic cells. This observation is partially related to the selective reduction of hemolysis while preserving antibacterial effects reported previously in AMP mixtures obtained by copolymerizing L-Lys and L-Phe upon switching to copolymerizing L-Lys and DPhe, ${ }^{7,8}$ although these polymers are made from enantiomerically pure amino acids, while we are using racemic amino acids.

Partial Stereorandomization of T25 Shows That $\alpha$ Helical Folding of the Dendrimer Core Triggers Hemolysis and Toxicity but Is Not Required for Antibacterial Effects. As for $\alpha$-helical AMPs and other AMPDs, the CD spectrum of the all L- enantiomer L-T25 features a strong $\alpha$-helical signal in the presence of TFE (Figure 3d, blue trace). Indeed, molecular dynamics (MD) simulation of AMPD L-T25 using GROMACS ${ }^{40}$ showed that an $\alpha$-helix involving the $\alpha$-peptide backbone spanning from the dendrimer core (G0) through the first generation (G1) and second generation (G2) branches should be stable and probably accounted for the $\alpha$-helical CD signal (Figure 3e, 
a)

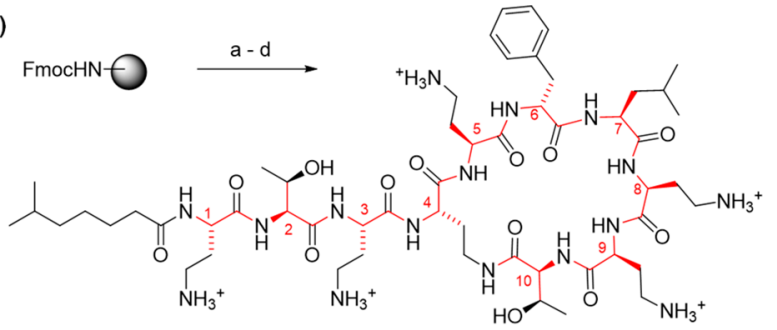

PMB2 (Polymyxin B2)

sr-PMB2 (all residues racemic)

$s r$-PMB2a (all racemic except 2, 5, 6, 8)

st-PMB2b (all racemic except 1, 5, 6, 7, 8) b)

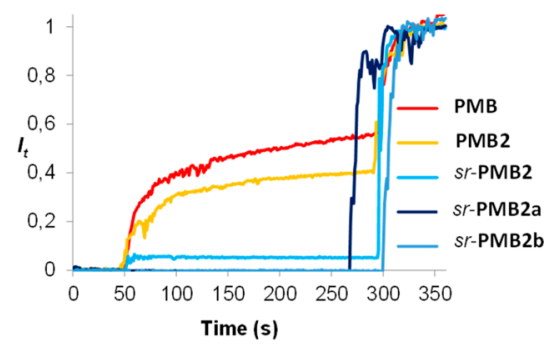

c)
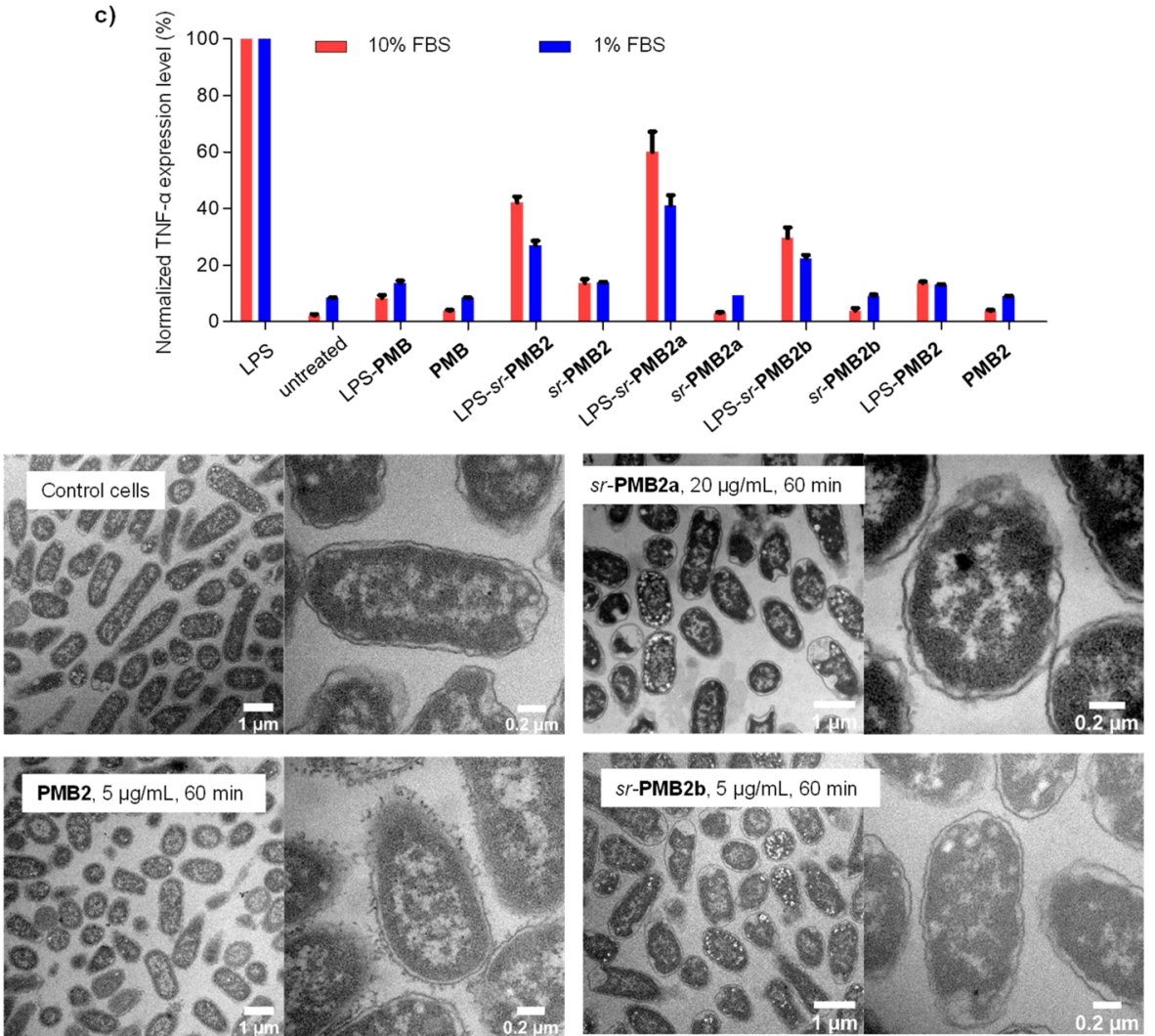

Figure 4. Stereorandomization of polymyxin B2. (a) Solid phase total synthesis of polymyxin B2. Conditions: a) linear chain synthesis by SPPS; b) on-resin cyclization: (i) $\mathrm{Pd}\left(\mathrm{Ph}_{3}\right)_{4}$ (0.25 equiv), $\mathrm{PhSiH}_{3}$ (25 equiv), in dry DCM, (ii) Fmoc deprotection with $20 \%$ v/v piperidine in DMF, (iii) Oxyma (7.5 equiv), DIC (10 equiv), $25{ }^{\circ} \mathrm{C}, 12 \mathrm{~h}$; c) TFA cleavage with TFA/TIS/ $\mathrm{H}_{2} \mathrm{O}$ (94:5:1), $4 \mathrm{~h}, 25{ }^{\circ} \mathrm{C}$, then HPLC purification. (b) Membrane-disruptive properties of commercial polymyxin and synthetic polymyxin B analogues (10 mM TRIS, $107 \mathrm{mM} \mathrm{NaCl}, \mathrm{pH} 7.4)$. After $50 \mathrm{~s}$ the indicated compound was added to reach the indicated concentration. After $300 \mathrm{~s} \mathrm{1.2 \%} \mathrm{Triton} \mathrm{X-100} \mathrm{was} \mathrm{added} \mathrm{for} \mathrm{full} \mathrm{fluorescein} \mathrm{release.} \mathrm{(c)}$ Determination of TNF- $\alpha$ expression level in Raw264.7 murine macrophages by ELISA immunoassay. Polymyxin derivatives were coincubated with or without $0.1 \mu \mathrm{g} / \mathrm{mL}$ of E. coli LPS containing 1 or $10 \% \mathrm{FBS}$ for $4 \mathrm{~h}$ at $37^{\circ} \mathrm{C}$ and $5 \% \mathrm{CO}_{2}$. (d) TEM images of E. coli cells treated for 60 min at 20 $\times$ MIC with polymyxin B analogues. see the Supporting Information for details.

Figure S18). Strikingly, the heptapeptide spanning through G0 and G1 formed an amphiphilic $\alpha$-helix exposing a compact hydrophobic patch of four leucine residues on the dendrimer surface (Figure 3f). To test whether the $\alpha$-helical conformation of the G1-G0 heptapeptide might be responsible for the hemolytic properties of L-T25, we prepared partially stereorandomized analogues featuring either a homochiral dendrimer core (G0 and G1) and stereorandomized outer branches (G2 and G3, sr-T25a), or vice versa ( $s r$-T25b, Table 1$)$.

Both $s r-\mathbf{T 2 5 a}$ and $s r$-T25b showed similar antibacterial, EYPG and EYPC vesicle leakage activities as L-T25 and $s r-T 25$ (Table 1, Figure 3b). Furthermore, only AMPD sr-T25a with the homochiral core showed an $\alpha$-helical CD spectrum similar to L-T25 (Figure 3d, red trace) and retained its strong hemolytic effect (Table 1). By contrast, analogue $s r$-T25b with a stereorandomized core and homochiral outer branches had a CD spectrum indicating a disordered conformation (Figure 3d, green trace), and completely suppressed EYPC vesicle leakage activity and hemolysis (Table 1 , Figure $3 \mathrm{~b}$ ).

Taken together, these data showed that the $\alpha$-helical core is responsible for the hemolytic activity and cell toxicity of L-T25, presumably mediated by formation of a hydrophobic patch of four leucine residues. The fact that $s r$-T25b largely preserves its antibacterial activity shows that the folded core is however not necessary in this case. 
Full or Partial Stereorandomization of the Cyclic Peptide Polymyxin B2 Suppresses Membrane Disruption and LPS Binding but Partially Preserves Antibacterial Activity. To test whether stereorandomization might also affect the activity of cyclic peptides, we investigated the last resort antibiotic PMB, which is also a membranedisruptive compound as the AMPs and AMPDs discussed above. $^{41,42}$ In addition, PMB binds strongly to lipid A and thereby neutralizes the immune activating properties of the lipopolysaccharide (LPS) released upon lysis of bacterial cells. ${ }^{43}$ Structure-activity relationship studies have identified residues critical for the activity of PMB. ${ }^{29,44}$ However, in terms of stereochemistry, only the LPS binding activity of the cyclic portion of PMB and its enantiomer has been investigated. ${ }^{45}$

Commercial PMB is a mixture of PMB1, acylated with 6methyl-octanoic acid at the $N$-terminus, and PMB2, acylated with 6-methyl-heptanoic acid at the $N$-terminus. Here we investigated PMB2, which we synthesized using the reported SPPS approach. ${ }^{38}$ We used the same method to obtain the fully stereorandomized ( $s r$-PMB2) and two partially stereorandomized analogues fixing stereochemistry in positions 2,5 , $6,8(s r$-PMB2a) or positions $1,5,6,7,8$ (sr-PMB2b), which have been previously reported to be critical for activity (Figure 4a). ${ }^{29,45}$

While our synthetic PMB2 had the similar activity as commercial PMB, sr-PMB2 essentially lost all of the antimicrobial and antibiofilm activity of the homochiral parent compound except against E. coli ( $\mathrm{MIC}=2 \mu \mathrm{g} / \mathrm{mL}$, Table 1). Vesicle leakage activity on EYPG vesicles, reflecting membrane-disruptive activity, was also abolished in $s r$-PMB2 (Figure 4b, Figure S14). Furthermore, sr-PMB showed reduced LPS neutralizing activity, as assessed by LPS induced TNF- $\alpha$ release from mouse macrophages (Figure $4 c$ ).

The partially stereorandomized analogues $s r$-PMB2a and $s r$ PMB2b also lost their membrane disruptive and, in part, their LPS neutralizing activities (Figure $4 \mathrm{~b}, \mathrm{c}$ ). However, both analogues retained significant antibacterial activity; in particular, sr-PMB2b had the same activity as PMB against $E$. coli $(\mathrm{MIC}=0.25 \mu \mathrm{g} / \mathrm{mL}$, Table 1$)$. Strikingly, TEM images of $E$. coli cells exposed to the different PMB analogues at $20 \times$ MIC showed that only PMB2 triggered detectable membrane damage on the bacteria, in line with the vesicle leakage activity data (Figure 4d, Figure S17). Strikingly, sr-PMB2a and $s r$ PMB2b did not induce any visible damage despite of their activity against these bacteria.

The different membrane-disruptive and antibacterial effects observed with PMB2 and its full or partially stereorandomized analogues demonstrate that stereochemical purity is essential for membrane disruption and LPS neutralization by this antibiotic but also point to the existence of alternative targets at least in the case of $E$. coli.

\section{CONCLUSIONS}

In summary, we have shown, at the example of antimicrobial peptides, peptide dendrimers, and the cyclic peptide polymyxin B2, that SPPS with racemic amino acids readily delivers $s r-$ analogues as well-defined homogeneous products. $s r$-Analogues exhibit distinct properties compared with the all $\mathrm{L}-$, all $\mathrm{D}-$, or rac- (1:1 mixture of all $\mathrm{L}^{-}$and all $\left.\mathrm{D}-\right)$ compounds, in particular specific modulation of membrane disruption, hemolysis and cytotoxicity. Comparing $s r$ - with all $\mathrm{L}^{-}$or all D-peptides generally offers a straightforward method to probe the role of stereochemical purity and folding in activity and potentially unravel diverse mechanisms of action involving different conformations of the same peptide. This new approach should be broadly applicable to better understand and improve the activity and properties of peptides for various types of activity.

\section{METHODS}

Synthesis and characterization of peptide dendrimers and all assays, measurements, and modeling studies are described in the Supporting Information. No unexpected or unusually high safety hazards were encountered.

\section{ASSOCIATED CONTENT}

\section{SI Supporting Information}

The Supporting Information is available free of charge at https://pubs.acs.org/doi/10.1021/acscentsci.0c01135.

Details of synthesis and characterization of peptide dendrimers and all assays, measurements, and modeling studies (PDF)

\section{AUTHOR INFORMATION}

\section{Corresponding Author}

Jean-Louis Reymond - Department of Chemistry and Biochemistry, University of Bern, 3012 Bern, Switzerland; (1) orcid.org/0000-0003-2724-2942; Email: jean-

louis.reymond@dcb.unibe.ch

\section{Authors}

Thissa N. Siriwardena - Department of Chemistry and Biochemistry, University of Bern, 3012 Bern, Switzerland

Bee-Ha Gan - Department of Chemistry and Biochemistry, University of Bern, 3012 Bern, Switzerland

Thilo Köhler - Department of Microbiology and Molecular Medicine, University of Geneva, Service of Infectious Diseases, University Hospital of Geneva, 1211 Geneva, Switzerland

Christian van Delden - Department of Microbiology and Molecular Medicine, University of Geneva, Service of Infectious Diseases, University Hospital of Geneva, 1211 Geneva, Switzerland

Sacha Javor - Department of Chemistry and Biochemistry, University of Bern, 3012 Bern, Switzerland

Complete contact information is available at: https://pubs.acs.org/10.1021/acscentsci.0c01135

\section{Author Contributions}

T.N.S. designed and carried out the study and wrote the paper. B.H.G. carried out TEM and cell culture experiments. T.K. and C.v.D. designed and supervised experiments with MDR bacteria. S.J designed and carried out molecular dynamics studies and wrote the paper. J.L.R. designed and supervised the study and wrote the paper. All authors read and commented the paper.

Notes

The authors declare no competing financial interest.

\section{ACKNOWLEDGMENTS}

This work was supported financially by the Swiss National Science Foundation (Grant nos. 200020_178998 and 407240_167048)

\section{REFERENCES}

(1) Merrifield, R. B. Solid Phase Peptide Synthesis. I. The Synthesis of a Tetrapeptide. J. Am. Chem. Soc. 1963, 85 (14), 2149-2154. 
(2) Amblard, M.; Fehrentz, J.-A.; Martinez, J.; Subra, G. Methods and Protocols of Modern Solid Phase Peptide Synthesis. Mol. Biotechnol. 2006, 33 (3), 239-254.

(3) Lam, K. S.; Salmon, S. E.; Hersh, E. M.; Hruby, V. J.; Kazmierski, W. M.; Knapp, R. J. A New Type of Synthetic Peptide Library for Identifying Ligand-Binding Activity. Nature 1991, 354 (6348), 8284.

(4) Komnatnyy, V. V.; Nielsen, T. E.; Qvortrup, K. Bead-Based Screening in Chemical Biology and Drug Discovery. Chem. Commun. 2018, 54 (50), 6759-6771.

(5) Schwaar, T.; Lettow, M.; Remmler, D.; Börner, H. G.; Weller, M. G. Efficient Screening of Combinatorial Peptide Libraries by Spatially Ordered Beads Immobilized on Conventional Glass Slides. High Throughput 2019, 8 (2). DOI: 10.3390/ht8020011.

(6) Houghten, R. A.; Pinilla, C.; Blondelle, S. E.; Appel, J. R.; Dooley, C. T.; Cuervo, J. H. Generation and Use of Synthetic Peptide Combinatorial Libraries for Basic Research and Drug Discovery. Nature 1991, 354 (6348), 84-86.

(7) Hayouka, Z.; Chakraborty, S.; Liu, R.; Boersma, M. D.; Weisblum, B.; Gellman, S. H. Interplay among Subunit Identity, Subunit Proportion, Chain Length, and Stereochemistry in the Activity Profile of Sequence-Random Peptide Mixtures. J. Am. Chem. Soc. 2013, 135 (32), 11748-11751.

(8) Amso, Z.; Hayouka, Z. Antimicrobial Random Peptide Cocktails: A New Approach to Fight Pathogenic Bacteria. Chem. Commun. 2019, 55 (14), 2007-2014.

(9) Durani, S. Protein Design with L- and d- $\alpha$-Amino Acid Structures as the Alphabet. Acc. Chem. Res. 2008, 41 (10), 13011308.

(10) Albada, H. B.; Prochnow, P.; Bobersky, S.; Bandow, J. E.; Metzler-Nolte, N. Highly Active Antibacterial Ferrocenoylated or Ruthenocenoylated Arg-Trp Peptides Can Be Discovered by an L-toD Substitution Scan. Chem. Sci. 2014, 5 (11), 4453-4459.

(11) Hazam, P. K.; Jerath, G.; Chaudhary, N.; Ramakrishnan, V. Peptido-Mimetic Approach in the Design of Syndiotactic Antimicrobial Peptides. Int. J. Pept. Res. Ther. 2018, 24 (2), 299-307.

(12) Nagy, K. J.; Giano, M. C.; Jin, A.; Pochan, D. J.; Schneider, J. P. Enhanced Mechanical Rigidity of Hydrogels Formed from Enantiomeric Peptide Assemblies. J. Am. Chem. Soc. 2011, 133 (38), 1497514977.

(13) Swanekamp, R. J.; DiMaio, J. T. M.; Bowerman, C. J.; Nilsson, B. L. Coassembly of Enantiomeric Amphipathic Peptides into Amyloid-Inspired Rippled $\beta$-Sheet Fibrils. J. Am. Chem. Soc. 2012, 134 (12), 5556-5559.

(14) Torbeev, V.; Grogg, M.; Ruiz, J.; Boehringer, R.; Schirer, A.; Hellwig, P.; Jeschke, G.; Hilvert, D. Chiral Recognition in Amyloid Fiber Growth. J. Pept. Sci. 2016, 22 (5), 290-304.

(15) Dutta, S.; Foley, A. R.; Warner, C. J. A.; Zhang, X.; Rolandi, M.; Abrams, B.; Raskatov, J. A. Suppression of Oligomer Formation and Formation of Non-Toxic Fibrils upon Addition of Mirror-Image A $\beta 42$ to the Natural l-Enantiomer. Angew. Chem., Int. Ed. 2017, 56 (38), $11506-11510$.

(16) Nguyen, L. T.; Haney, E. F.; Vogel, H. J. The Expanding Scope of Antimicrobial Peptide Structures and Their Modes of Action. Trends Biotechnol. 2011, 29 (9), 464-472.

(17) Mojsoska, B.; Jenssen, H. Peptides and Peptidomimetics for Antimicrobial Drug Design. Pharmaceuticals 2015, 8 (3), 366-415.

(18) Torres, M. D. T.; Sothiselvam, S.; Lu, T. K.; de la FuenteNunez, C. Peptide Design Principles for Antimicrobial Applications. J. Mol. Biol. 2019, 431, 3547.

(19) Neundorf, I. Antimicrobial and Cell-Penetrating Peptides: How to Understand Two Distinct Functions Despite Similar Physicochemical Properties. Adv. Exp. Med. Biol. 2019, 1117, 93-109.

(20) Stach, M.; Maillard, N.; Kadam, R. U.; Kalbermatter, D.; Meury, M.; Page, M. G. P.; Fotiadis, D.; Darbre, T.; Reymond, J.-L. Membrane Disrupting Antimicrobial Peptide Dendrimers with Multiple Amino Termini. MedChemComm 2012, 3 (1), 86-89.

(21) Stach, M.; Siriwardena, T. N.; Kohler, T.; van Delden, C.; Darbre, T.; Reymond, J. L. Combining Topology and Sequence
Design for the Discovery of Potent Antimicrobial Peptide Dendrimers against Multidrug-Resistant Pseudomonas Aeruginosa. Angew. Chem., Int. Ed. 2014, 53 (47), 12827-12831.

(22) Pires, J.; Siriwardena, T. N.; Stach, M.; Tinguely, R.; Kasraian, S.; Luzzaro, F.; Leib, S. L.; Darbre, T.; Reymond, J. L.; Endimiani, A. In Vitro Activity of the Novel Antimicrobial Peptide Dendrimer G3KL against Multidrug-Resistant Acinetobacter Baumannii and Pseudomonas Aeruginosa. Antimicrob. Agents Chemother. 2015, 59 (12), 7915-7918.

(23) Abdel-Sayed, P.; Kaeppeli, A.; Siriwardena, T.; Darbre, T.; Perron, K.; Jafari, P.; Reymond, J. L.; Pioletti, D. P.; Applegate, L. A. Anti-Microbial Dendrimers against Multidrug-Resistant P. Aeruginosa Enhance the Angiogenic Effect of Biological Burn-Wound Bandages. Sci. Rep. 2016, 6, 1-10.

(24) Siriwardena, T. N.; Stach, M.; He, R.; Gan, B.-H.; Javor, S.; Heitz, M.; Ma, L.; Cai, X.; Chen, P.; Wei, D.; Li, H.; Ma, J.; Köhler, T.; van Delden, C.; Darbre, T.; Reymond, J.-L. Lipidated Peptide Dendrimers Killing Multidrug-Resistant Bacteria. J. Am. Chem. Soc. 2018, 140 (1), 423-432.

(25) Siriwardena, T. N.; Capecchi, A.; Gan, B. H.; Jin, X.; He, R.; Wei, D.; Ma, L.; Kohler, T.; van Delden, C.; Javor, S.; Reymond, J. L. Optimizing Antimicrobial Peptide Dendrimers in Chemical Space. Angew. Chem., Int. Ed. 2018, 57 (28), 8483-8487.

(26) Siriwardena, T. N.; Lüscher, A.; Köhler, T.; van Delden, C.; Javor, S.; Reymond, J.-L. Antimicrobial Peptide Dendrimer Chimera. Helv. Chim. Acta 2019, 102 (4), e1900034.

(27) Gan, B.-H.; Siriwardena, T. N.; Javor, S.; Darbre, T.; Reymond, J.-L. Fluorescence Imaging of Bacterial Killing by Antimicrobial Peptide Dendrimer G3KL. ACS Infect. Dis. 2019, 5 (12), 2164-2173.

(28) Jeddou, F. B.; Falconnet, L.; Luscher, A.; Siriwardena, T.; Reymond, J.-L.; van Delden, C.; Köhler, T. Adaptive and Mutational Responses to Peptide Dendrimer Antimicrobials in Pseudomonas Aeruginosa. Antimicrob. Agents Chemother. 2020, 64 (4), DOI: 10.1128/AAC.02040-19.

(29) Velkov, T.; Thompson, P. E.; Nation, R. L.; Li, J. StructureActivity Relationships of Polymyxin Antibiotics. J. Med. Chem. 2010, 53 (5), 1898-1916.

(30) Baeriswyl, S.; Gan, B.-H.; Siriwardena, T. N.; Visini, R.; Robadey, M.; Javor, S.; Stocker, A.; Darbre, T.; Reymond, J.-L. X-Ray Crystal Structures of Short Antimicrobial Peptides as Pseudomonas Aeruginosa Lectin B Complexes. ACS Chem. Biol. 2019, 14 (4), 758766.

(31) Jasanoff, A.; Fersht, A. R. Quantitative Determination of Helical Propensities from Trifluoroethanol Titration Curves. Biochemistry 1994, 33 (8), 2129-2135.

(32) Arunkumar, A. I.; Kumar, T. K.; Yu, C. Specificity of HelixInduction by 2,2,2-Trifluoroethanol in Polypeptides. Int. J. Biol. Macromol. 1997, 21 (3), 223-230.

(33) de la Fuente-Nunez, C.; Reffuveille, F.; Mansour, S. C.; Reckseidler-Zenteno, S. L.; Hernandez, D.; Brackman, G.; Coenye, T.; Hancock, R. E. D-Enantiomeric Peptides That Eradicate WildType and Multidrug-Resistant Biofilms and Protect against Lethal Pseudomonas Aeruginosa Infections. Chem. Biol. 2015, 22 (2), 196205.

(34) Selsted, M. E.; Novotny, M. J.; Morris, W. L.; Tang, Y. Q.; Smith, W.; Cullor, J. S. Indolicidin, a Novel Bactericidal Tridecapeptide Amide from Neutrophils. J. Biol. Chem. 1992, 267 (7), 4292-4295.

(35) Falla, T. J.; Karunaratne, D. N.; Hancock, R. E. W. Mode of Action of the Antimicrobial Peptide Indolicidin. J. Biol. Chem. 1996, 271 (32), 19298-19303.

(36) Sitaram, N.; Subbalakshmi, C.; Nagaraj, R. Indolicidin, a 13Residue Basic Antimicrobial Peptide Rich in Tryptophan and Proline, Interacts with $\mathrm{Ca} 2+-\mathrm{Calmodulin}$. Biochem. Biophys. Res. Commun. 2003, 309 (4), 879-884.

(37) Hsu, C.-H.; Chen, C.; Jou, M.-L.; Lee, A. Y.-L.; Lin, Y.-C.; Yu, Y.-P.; Huang, W.-T.; Wu, S.-H. Structural and DNA-Binding Studies on the Bovine Antimicrobial Peptide, Indolicidin: Evidence for 
Multiple Conformations Involved in Binding to Membranes and DNA. Nucleic Acids Res. 2005, 33 (13), 4053-4064.

(38) Xu, W.-L.; Cui, A.-L.; Hu, X.-X.; You, X.-F.; Li, Z.-R.; Zheng, J.S. A New Strategy for Total Solid-Phase Synthesis of Polymyxins. Tetrahedron Lett. 2015, 56 (33), 4796-4799.

(39) Hennig, A.; Gabriel, G. J.; Tew, G. N.; Matile, S. StimuliResponsive Polyguanidino-Oxanorbornene Membrane Transporters as Multicomponent Sensors in Complex Matrices. J. Am. Chem. Soc. 2008, 130 (31), 10338-10344.

(40) Abraham, M. J.; Murtola, T.; Schulz, R.; Páll, S.; Smith, J. C.; Hess, B.; Lindahl, E. GROMACS: High Performance Molecular Simulations through Multi-Level Parallelism from Laptops to Supercomputers. SoftwareX 2015, 1-2, 19-25.

(41) Deris, Z. Z.; Swarbrick, J. D.; Roberts, K. D.; Azad, M. A. K.; Akter, J.; Horne, A. S.; Nation, R. L.; Rogers, K. L.; Thompson, P. E.; Velkov, T.; Li, J. Probing the Penetration of Antimicrobial Polymyxin Lipopeptides into Gram-Negative Bacteria. Bioconjugate Chem. 2014, 25 (4), 750-760.

(42) Berglund, N. A.; Piggot, T. J.; Jefferies, D.; Sessions, R. B.; Bond, P. J.; Khalid, S. Interaction of the Antimicrobial Peptide Polymyxin B1 with Both Membranes of E. Coli: A Molecular Dynamics Study. PLoS Comput. Biol. 2015, 11 (4), e1004180.

(43) Harm, S.; Gabor, F.; Hartmann, J. Low-Dose Polymyxin: An Option for Therapy of Gram-Negative Sepsis. Innate Immun. 2016, 22 (4), 274-283.

(44) Kanazawa, K.; Sato, Y.; Ohki, K.; Okimura, K.; Uchida, Y.; Shindo, M.; Sakura, N. Contribution of Each Amino Acid Residue in Polymyxin $\mathrm{B}_{3}$ to Antimicrobial and Lipopolysaccharide Binding Activity. Chem. Pharm. Bull. 2009, 57 (3), 240-244.

(45) Tsubery, H.; Ofek, I.; Cohen, S.; Fridkin, M. The Functional Association of Polymyxin B with Bacterial Lipopolysaccharide Is Stereospecific: Studies on Polymyxin B Nonapeptide. Biochemistry 2000, 39 (39), 11837-11844. 